Charles Theillet Philippe Jeanteur

\section{Anomalies génomiques somatiques et cancer du sein}

Du fait de son importance économique et sociale (c'est, en Europe de l'Ouest et en Amérique du Nord, la première cause de mortalité par cancer chez la femme), l'étude du cancer du sein est un objectif prioritaire. Son évolution s'accompagne d'un catalogue complet d'anomalies puisqu'on y recense des amplifications d'ADN sur au moins cinq régions chromosomiques distinctes et des pertes de matériel génétique impliquant dix autres sites chromosomiques, auxquelles on peut ajouter l'inactivation par mutation ponctuelle de l'anti-oncogène $p 53$. Les combinaisons d'amplifications d'oncogènes et de pertes $d$ 'anti-oncogènes sont donc multiples, et la détermination de leur ordre d'apparition pourrait permettre de mieux connaître et comprendre les différentes étapes de la progression tumorale de ce cancer.

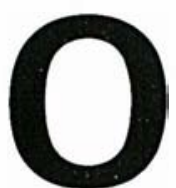

n peut désormais considérer comme acquis que l'origine du cancer se trouve dans nos gènes. L'association entre développement, progression tumorale et accumulation d'anomalies au niveau de l'ADN est un phénomène observé de longue date. Ces mutations somatiques touchent probablement une grande variété de gènes, mais certains auront une importance toute particulière. En effet, l'altération d'un protooncogène ou d'un anti-oncogène aura des effets dévastateurs, du fait du rôle de ces gènes dans le maintien de l'équilibre délicat, au sein d'un tissu, entre prolifération, différenciation et mort cellulaire. La mutation d'un proto-oncogène provoquera son activation en oncogène, il s'agira d'un gain de fonction agissant sur un mode dominant positif. Dans le cas d'un gène suppresseur de tumeur, on assiste à une perte de fonction et ces mutations sont généralement considérées comme récessives, bien que certains éléments récents suggèrent l'existence de mécanismes dominants négatifs. La conséquence sera l'inactivation d'un régulateur négatif de la prolifération cellulaire. Ces muta- 


\section{RÉFÉRENCES}

1. Szepetowski P, Perucca-Lostanlen D, Gaudray P. Amplification, proto-oncogènes et cancers humains. médecine/sciences 1991 ; 7: 14-21.

2. Gaudray P, Szepetowski P, Escot C, Birnbaum D, Theillet C. DNA amplification at 11 q13 in human cancer : from complexity to perplexity. Mutation Res 1992 ; $276: 317-29$

3. Tlsty TD, White A, Sanchez J. Suppression of gene amplification in human cell hybrids. Science 1992 ; 255 : 1425-7.

4. Gullick WJ. The role of the epidermal growth factor receptor- and the c-erB-2 protein in breast cancer. Int J Cancer $1990 ; 5$ : 55-61

5. Holmes WE, Sliwkowski MX, Akita $\mathrm{RW}$, et al. Identification of heregulin, a specific activator of p185 crbB2. Science 1992 ; 256 : 1205-10.

6. Wen D, Peles E, Cupples R, et al. Neu differentiation factor: a transmembrane glycoprotein containing an EGF domain and an immunoglobulin homology unit. Cell $1992 ; 69$ : 559-72.

7. Peles E, Bacus SS, Koski RA, et al. Isolation of the Neu/HER-2 stimulatory ligand: a $44 \mathrm{kd}$ glycoprotein that induces differentiation of mammary tumor cells. Cell 1992 ; 69 : 205-16.

8. Slamon DK, Clark GM, Wong SG, Levin WJ, Ullrich A, McGuire WL. Human breast cancer : correlation of relapse and survival with amplification of the HER-2/neu oncogene. Science 1987; 235 : 177-82.

9. Borg A, Baldetorp B, Ferno M, Killander $D$, Olsson $H$, Sigurdsson $H$. erbB2 amplification in breast cancer with a high rate of proliferation. Oncogene 1991; 6 : $137-43 ; 50$ (14) : 4332-7.

10. Van de Vijuer MJ, Peterse JL, Mooi WJ, et al. Neu-protein overexpression in breast cancer. Association with comedotype ductal carcinoma in situ and limited prognostic value in stage II breast cancer. $N$ Engl J Med 1988 ; 319 : 1239-45.

11. Lammie AG, Peters G. Chromosome $11 \mathrm{q} 13$ abnormalities in human cancer. Cancer Cells $1991 ; 3$ : 413-20.

12. Peters G. Oncogenes at viral integra- tions, au sens large du terme, vont de simples substitutions nucléotidiques, addition ou perte de quelques nucléotides, à des réarrangements plus importants tels que des amplifications de l'ADN, des translocations et des délétions chromosomiques, ou encore des insertions mutagènes de génomes viraux ou d'éléments génétiques mobiles.

On voit donc que la prolifération maligne résulte (ou s'accompagne) de combinaisons d'altérations génétiques, touchant des proto-oncogènes et des anti-oncogènes, et on peut logiquement penser que ces anomalies agissent en synergie. Les altérations génétiques semblent apparaître successivement et être cumulatives, mais l'ordre dans lequel elles surviennent peut varier et les combinaisons d'activations d'oncogènes et de pertes d'anti-oncogènes sont diverses. Par ailleurs, on peut rencontrer les mêmes associations dans des tumeurs d'origines très différentes, alors que certaines altérations semblent restreintes à des types tumoraux particuliers. Une analyse systématique des anomalies génomiques permet d'envisager l'établissement de "cartes" génétiques des cancers. Bien que tous les gènes impliqués dans les cancers ne soient pas actuellement connus, et donc qu'il ne soit pas encore possible de reconstituer l'" histoire moléculaire " de chaque tumeur, ni a fortiori d'agir directement sur les molécules responsables de la prolifération, l'analyse moléculaire a d'ores et déjà trois buts : (1) continuer à identifier les cibles de ces altérations qui sont autant de clés vers la compréhension des mécanismes de l'oncogenèse ; (2) organiser le dépistage des facteurs de prédisposition héréditaires, tels que les altérations constitutives d'antioncogènes ou les allèles rares de proto-oncogènes ; (3) utiliser des marqueurs moléculaires dans l'évaluation pronostique et la classification des tumeurs, au même titre que les paramètres cliniques et anatomopathologiques. L'exemple du cancer du sein, développé dans cette revue, est, à cet égard, significatif et instructif.

\section{Activation d'oncogènes}

Des différents types d'anomalies génomiques pouvant provoquer l'acti- vation d'un proto-oncogène en oncogène c'est, dans le cancer du sein, l'amplification qui l'emporte largement tant par sa fréquence que par la diversité de gènes qui la subissent. L'amplification d'un gène, c'est-àdire l'augmentation du nombre de ses copies, au-delà des deux normalement présentes dans toute cellule somatique, est un phénomène sélectif qui semble particulier aux cellules cancéreuses. L'augmentation du nombre de copies d'un gène ayant en général pour corollaire une élévation de son niveau d'expression, elle conférera au clone cellulaire qui en sera porteur un avantage de croissance lui permettant d'émerger., On voit donc le bénéfice que pept tirer une tumeur de l'amplification d'un oncogène lui garantissant l'expression à de très hauts niveaux d'un gène stimulant la prolifération cellulaire. La relation entre amplification et cancers humains a été traitée, dans médecine/sciences, de façon plus extensive par Szepetowski et al. [1].

Amplifications géniques et relations avec l'évolution de la maladie Le cancer du sein semble particulièrement enclin à l'amplification d'ADN et on a déjà identifié à ce jour cinq gènes ou régions chromosomiques amplifiées dans les tumeurs sporadiques du sein (Tableau I). La fréquence de ce type d'événement y est telle que la moitié des tumeurs présentent au moins une amplification à un de ces loci [2]. A ce propos, on remarquera que la capacité à amplifier a été reliée à une instabilité génomique typiquement rencontrée dans des cellules tumorales, et il a été récemment démontré qu'elle était perdue dans des hybrides issus de la fusion de cellules normales et tumorales. Il semble donc que l'amplification d'ADN dépende d'un déterminisme récessif puisque les hybrides ne réacquièrent leur caractère amplificateur qu'après perte chromosomique $\left(\mathrm{m} / \mathrm{s} \quad n^{\circ} 5\right.$, vol. 8, p. 500) [3]. Enfin on notera que, dans le cancer du sein, à l'amplification d'oncogènes patentés, tels myc et erbB2 (aussi appelé neu) s'ajoute celle de régions chromosomiques où l'identification des gènes significatifs est plus complexe. Ce point sera développé plus bas. 
Tableau I

GĖNES OU RÉGIONS CHROMOSOMIQUES AMPLIFIÉES DANS LES TUMEURS DU SEIN

\begin{tabular}{|c|c|c|c|c|}
\hline Amplification & Fréquence & $\begin{array}{l}\text { Localisation } \\
\text { chromosomique }\end{array}$ & Corrélation & $\begin{array}{l}\text { Évolution probable } \\
\text { de la maladie }\end{array}$ \\
\hline myc & $16 \%$ & $8 p 24$ & Tumeurs de haut grade & Récidives rapides \\
\hline erbB2 & $21 \%$ & $17 q 11.2$ & Perte des récepteurs hormonaux & Récidive et décès accélérés \\
\hline $\begin{array}{l}\text { cycD1 } \\
\text { (bcl1/hst/int2) }\end{array}$ & $15 \%$ & $11 q 13$ & $\begin{array}{l}\text { Présence des récepteurs hormonaux } \\
\text { Envahissement des ganglions axillai- } \\
\text { res }\end{array}$ & $\begin{array}{l}\text { Récidives à moyen et long } \\
\text { terme }\end{array}$ \\
\hline$f / g ?$ & $12 \%$ & $8 p 12$ & Amplification $11 \mathrm{q} 13$ & $?$ \\
\hline bek? & $12 \%$ & $10 q 24$ & Amplification myc & $?$ \\
\hline
\end{tabular}

- Le gène $c-m y c$ agit comme un régulateur central dans la prolifération et la différenciation cellulaire et se trouve être fréquemment la cible d'anomalies génomiques dans des cancers de types variés, amplification dans le cancer du poumon ou réarrangement dans les lymphomes de Burkitt et les leucémies lymphocytaires aiguës. Les fréquences d'amplification rapportées dans les tumeurs du sein varient de 6 à $40 \%$ selon les auteurs, mais la plupart s'accordent pour relier l'amplification et la surexpression de myc aux tumeurs les plus agressives, encore que les relations avec les paramètres cliniques et histopathologiques soient parfois controversées [1]. En effet celles-ci ont été, selon les études, corrélées significativement à l'apparition de récidives, à l'envahissement des ganglions axillaires ou aux tumeurs de haut grade (Tableau I) [2].

- Le gène erbB2, initialement identifié sous le nom de neu dans des neuroblastomes chimio-induits chez le rat (pour revue, voir [4]), code pour un récepteur membranaire à activité tyrosine kinase étroitement apparenté au récepteur de l'EGF codé par erbB1. Bien que l'activation de l'activité tyrosine kinase d'erbB2 ait été rapidement reliée à une augmentation de la prolifération cellulaire, le ligand de ce récepteur membranaire s'est longtemps fait attendre. Très récemment, deux groupes ont indépendamment décrit l'isolement d'un polypeptide d'environ $45 \mathrm{kDa}$ se liant spécifiquement à erbB2 et provoquant l'activation de son activité tyrosine kinase $\left([5,6], \quad m / s \quad n^{\circ} 7, \quad\right.$ vol. 8 , p. 734). Les séquences des clones ADNc montrent qu'il s'agit d'une famille de quatre facteurs [6] dont l'un induirait la différenciation de certaines lignées de cellules cancéreuses du sein [7]. La mise en évidence de ces ligands permettra de répondre à de nombreuses questions sur la régulation et les effets biologiques d'erbB2. De plus, on peut envisager le développement de voies thérapeutiques fondées sur l'utilisation d'antagonistes ou d'analogues de ces ligands.

C'est l'étude de Slamon et al. [8], montrant que $25 \%$ des tumeurs primitives analysées présentaient une amplification d'erbB2, et que cet événement était relié à l'envahissement des ganglions axillaires et à une augmentation des risques de rechutes et de mortalité, qui lança la mode erbB2/neu dans le cancer du sein. En quatre ans, on ne compte pas moins d'une centaine d'études, qui confirmant, qui infirmant les conclusions de Slamon. On note néanmoins que seule une minorité d'entre elles excluent tout lien entre l'amplification et/ou la surexpression du gène erbB2 et la gravité tumorale [4]. En effet, la plupart des auteurs observent une corrélation positive avec des paramètres clinico-pathologiques de mauvais pronostic, tels que l'envahissement ganglionnaire, d'autres constatent une forte liaison avec l'absence de récepteurs hormonaux (Tableau I), d'autres encore avec le grade histopathologique [4]. On relève aussi une relation avec la surexpression du récepteur de l'EGF et, plus récemment, avec l'aneuploïdie et un fort index de prolifération [2]. Le consensus qui semble se dégager actuellement est que l'amplification du gène erbB2 est significativement plus élevée dans les tumeurs ayant perdu les récepteurs aux œstrogènes $(\mathrm{RE}-)$ et/ou à la progestérone $(\mathrm{RP}-)$ et présentant une fraction aneuploïde assez importante. Cette corrélation entre amplification de erbB2 et absence de récepteurs aux hormones stéroïdes a soulevé l'hypothèse intéressante d'un passage, lors de la progression tumorale, d'un état d'hormono-sensibilité vers celui d'une autonomie de croissance rendue possible par la présence conjuguée des facteurs de croissance et de leurs récepteurs.

Concernant les relations avec le devenir du malade, il semble désormais raisonnable de considérer que l'amplification et/ou la surexpression d'erbB2 sont prédictives d'une évolution défavorable de la maladie [4]. Malgré son index élevé, la valeur pronostique de la positivité erbB2 reste cependant inférieure à celle de l'envahissement ganglionnaire. Elle est de plus atténuée, dans les cancers invasifs, par sa forte corrélation (donc sa redondance) avec la valeur péjorative de l'absence de récepteurs et par sa restriction aux patientes $\mathrm{N}+$.

Partant des constatations faites sur des cancers invasifs, l'attention s'est tournée vers les tumeurs in situ, généralement considérées comme des stades précédant l'invasivité. Ainsi, il est rapidement apparu qu'une forte proportion (70\% environ) de comédocarcinomes surexprimaient la protéine p185-erbB2 [10], ce qui représente 


\section{RÉFÉRENCES}

13. Szepetowski P, Courseaux A, Carle G Theillet C, Gaudray P. Amplification of 11 13 DNA sequences in human breast cancer : D11S97 identifies a region tightly linked to BCL1 which can be amplified separately. Oncogene $1992 ; 7$ : 751-5.

14. Kornbluth S, Paulson KE, Hanafusa $H$. Novel tyrosine kinase identified by phosphotyrosine antibody screening of cDNA libraries. Mol Cell Biol 1988 ; 8 : 5541-4.

15. Ruta M, Howk R, Ricca G, et al. A novel protein tyrosine kinase gene whose expression is modulated during endothelia cell differentiation. Oncogene $1988 ; 3$ : 9-15.

16. Adnane J, Gaudray P, Dionne C, et al. $B E K$ and $F L G$, two receptors to members of the FGF family are amplified in subsets of human breast cancers. Oncogene $1991 ; 6$ : 659-63.

17. Saint-Ruf C, Gerbeault-Seureau $M$ Viegas-Péquinot E, Zafrani B, Cassingena R, Dutrillaux B. Proto-oncogene amplification and homogeneously staining regions in human breast carcinomas. Genes Chrom Cancer $1990 ; 2$ : 18-26.

18. Berns EMJJ, Klijn JGM, Van Staveren IL, Portengen H, Foekens JA. Sporadic amplification of the insulin-like growth factor 1 receptor gene in human breast tumors. Cancer Res 1992 ; 52 : 1036-9.

20. Weinberg RA. Tumor suppressor genes. Science 1991; 254：1138-1146.

21. Callahan R, Campbell G. Mutations in human breast cancer : an overview. I Natl Cancer Inst 1989; $81: 1780-6$.

22. Junien C, Henry I. Empreinte parentale différentielle et cancer. médecine/sciences 1989 ; 7 (5) : 480-8.

23. Devilee $\mathrm{P}$, Van Vliet $\mathrm{M}$, Van Sloun $\mathrm{P}$, et al. Allelotype of human breast carcinoma : a second major site for loss of heterozygosity is on chromosome 6q. Oncogene 1991 ; 6 : 1705-11.

24. Cropp CS, Lidereau R, Campbell G, Champene MH, Callahan R. Loss of heterozygosity on chromosomes 17 and 18 in breast carcinoma : two additional regions identified. Proc Natl Acad Sci USA 1990 ; 87 :

une incidence trois fois supérieure à celle rencontrée dans les cancers invasifs. Ces résultats ont été confirmés par des études complémentaires et étendus aux autres types de tumeurs canalaires in situ dans lesquelles on détecte aussi des positifs pour la p185-erbB2, avec des fréquences toutefois inférieures à celles rencontrées dans les comédo-carcinomes [4]. Enfin, si l'on considère la protéine erbB2 comme une cible thérapeutique potentielle, le statut erbB2 d'une patiente prend toute son importance. En effet, plusieurs équipes travaillent sur des stratégies d'immunotoxines, prenant la portion extramembranaire pour cible, ou cherchent à évaluer chez l'animal l'effet de l'injection de récepteurs erbB2 solubles sur la croissance de cellules tumorales surexprimant la p185-erbB2.
- L'amplification de la région chromosomique 11q13 a d'abord été détectée avec la sonde int2 (pour revue, voir $[2,11])$ dont l'implication dans la carcinogenèse mammaire viro-induite chez la souris [12] laissait enfin entrevoir d'intéressants et logiques parallèles entre le système murin et le cancer du sein chez la femme. Rapidement, il est apparu que la région amplifiée englobait le gène hst ainsi que le locus bol1, qui définit par ailleurs une région chromosomique fréquemment impliquée dans les translocations $\mathrm{t}(11 ; 14)$ de certains lymphomes folliculaires $[2$, 11].

La co-amplification de hst, de int 2 et de $b c l 1$ définissait un domaine amplifié d'au moins $500 \mathrm{~kb}$. Cette taille étant compatible avec la présence de plusieurs gènes, l'identité réelle du

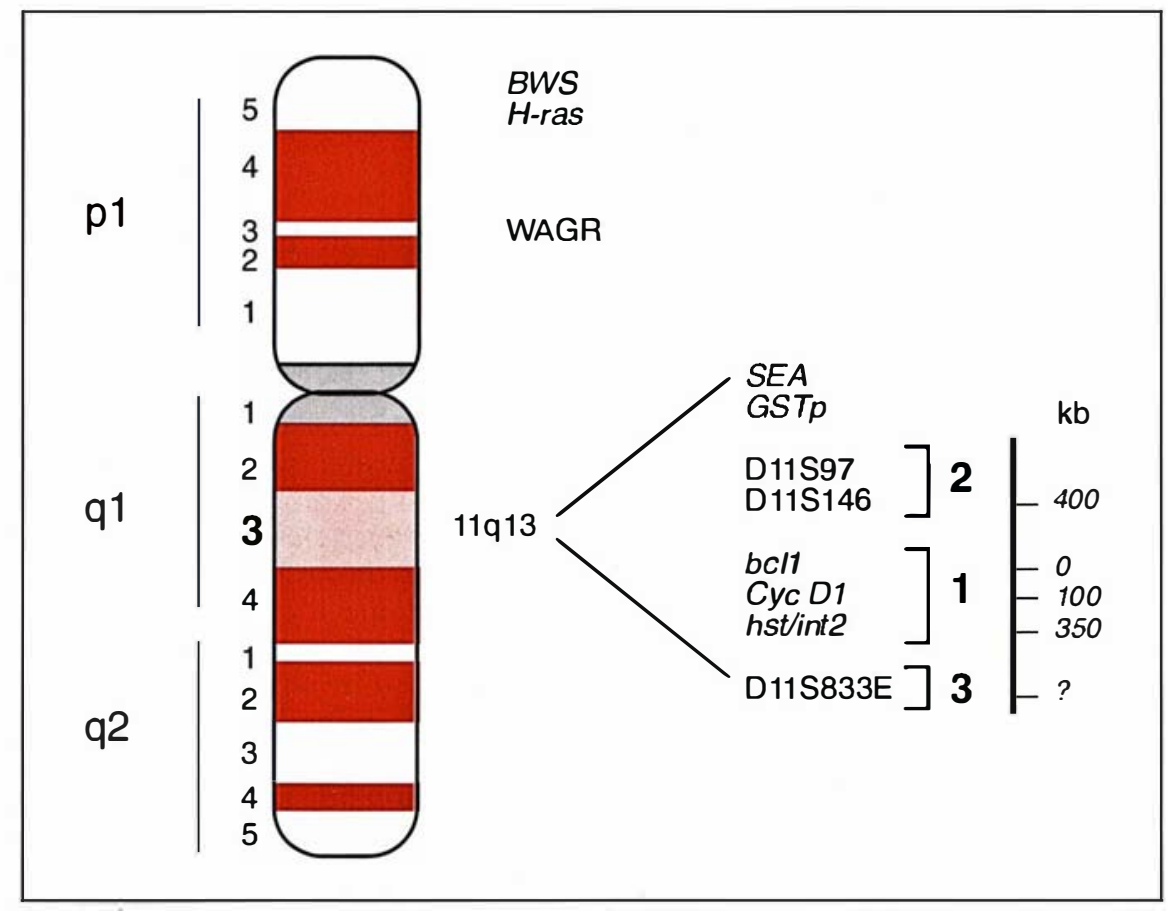

Figure 1. Carte du chromosome 11 humain et détail de la région amplifiée sur la bande 11q13. Dans l'éclaté sont présentés dans l'ordre de leur localisation, du centromère (haut) vers le télomère (bas), sur le chromosome les gènes ou les marqueurs significatifs. Les crochets et des numéros schématisent l'existence de groupes de marqueurs définissant une région amplifiée cohérente. L'échelle à droite indique les distances approximatives les séparant. SEA : S13 avian erythroblastosis oncogene homolog. GSTp : glutathione $S$ transferase 3 (pi). BCL1: B cell CLL/lymphoma 1. CYCDI : cyclin DI. HST : human stomach cancer. INT2 : MMTV integration site 2 homolog. BWS : Beckwith Wiedemann syndrome. WAGR : Wilms, Anaridia, Genito-Urinary, Retarded. 


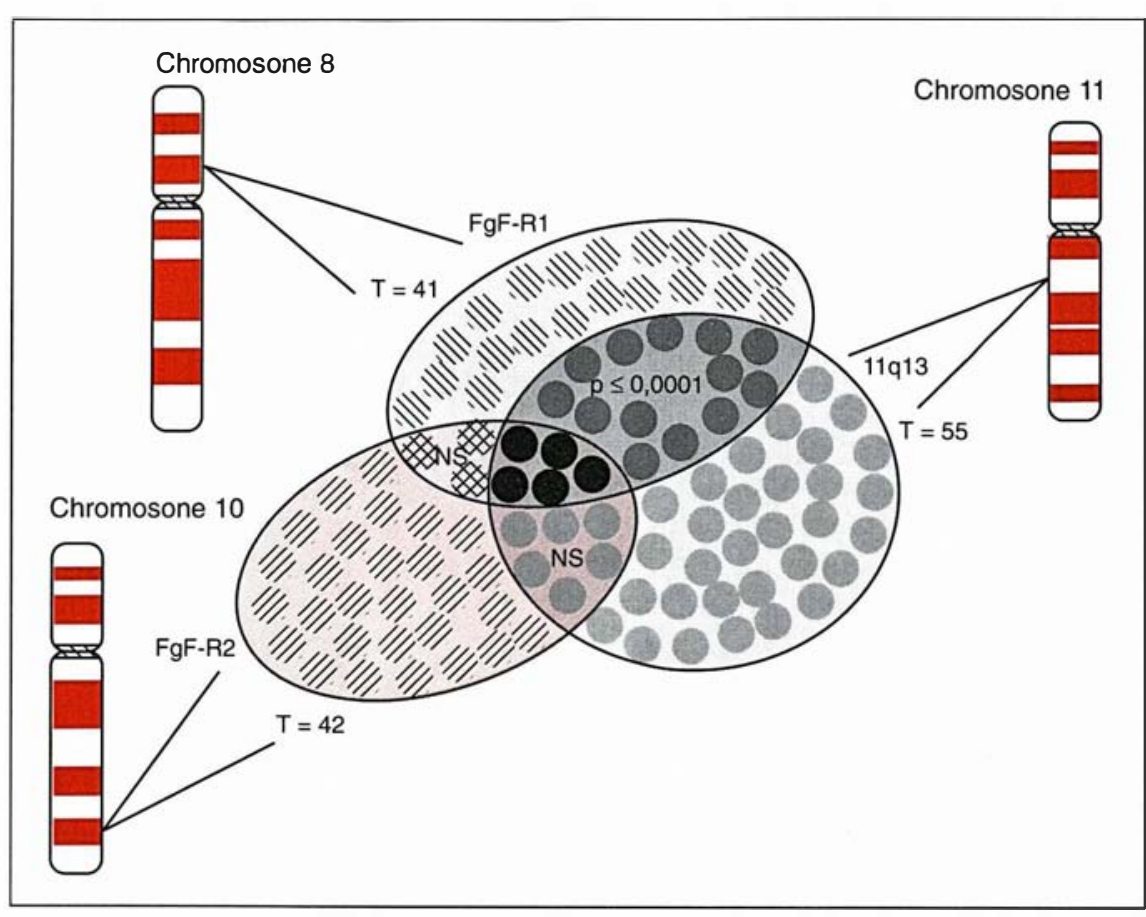

Figure 2. Interrelations entre les amplifications des régions 11q13, 10q26 et $8 p 12$ dans les cancers du sein. Chaque ovale représente un groupe de tumeurs présentant une amplification pour l'une des trois régions citées, les intersections correspondent aux tumeurs amplifiées pour deux, voire trois marqueurs et on note l'important recouvrement entre amplification en $8 p 12$ et $11 q 13$.

gène significatif dans cette amplification, c'est-à-dire celui qui confère l'avantage sélectif, resta longtemps inconnue. Ce n'est que récemment qu'un candidat sérieux à ce rôle a été identifié. Il s'agit du gène $c y c D 1$ codant pour le premier représentant des cyclines D. Il est fréquemment réarrangé dans les adénomes de la thyroïde, et systématiquement amplifié et surexprimé dans les tumeurs du sein où bcl1 est amplifié. $c y c D 1$ pourrait aussi être le gène activé dans les translocations $\mathrm{t}(11 ; 14)$ impliquant bcl1 $[2,11]$. Enfin, pour ajouter encore à la complexité de la situation, des travaux récents du groupe de P. Gaudray (Nice, France) ont montré que $c y c D 1$ n'était pas le seul foyer d'amplification dans la région $11 \mathrm{q} 13$ (figure 1) [13].

Quoi qu'il en soit, cette amplification détectée dans 30 à $50 \%$ des cancers ORL et $13 \%$ des cancers du poumon pourrait se révéler un marqueur intéressant dans le cancer du sein, où on la retrouve dans $15 \%$ des cas. Plusieurs études indépendantes ont en effet conclu que l'amplification de $\mathrm{m} / \mathrm{s} n^{\circ} 9$ vol. 8 , novembre 92 autres régions du génome où l'on observe des amplifications dans les cancers du sein et de l'ovaire. Initialement ces amplifications ont été détectées respectivement par les sondes des gènes $f g f R 1$ (appelé aussi $f l g$ ) et $f g f R 2$ (appelé aussi bek ou $K$-sam) $[14,15]$. Ces deux gènes codant pour des récepteurs membranaires à activité tyrosine kinase et leurs ligands appartenant à la famille des FGF (facteurs de croissance des fibroblastes), il pouvait être intéressant de tester une éventuelle corrélation dans l'amplification de ces deux partenaires potentiels. En fait, $f g f R 1$ et $f g f R 2$ sont amplifiés dans des populations de tumeurs distinctes et représentent donc des événements indépendants [16]. L'amplification de 8p12 s'apparente à celle de $11 \mathrm{q} 13$ en ce qui concerne les corrélations avec les paramètres cliniques. Fréquente dans les tumeurs ayant conservé les récepteurs hormonaux, elle est aussi significativement reliée à l'envahissement ganglionnaire. Ces tendances indiquent que les amplifications en 8 p12 et 11 q13 concernent des sous-populations tumorales similaires. De fait, les deux événements sont liés (Tableau I) puisqu'une proportion non négligeable de tumeurs amplifiées en 8p12 le sont aussi en $11 \mathrm{q} 13$ (figure 2). En revanche, rien de semblable n'est à remarquer en ce qui concerne l'amplification en 10q26 [2, 11].

Ce descriptif qui ne porte que sur cinq régions amplifiées, n'exclut pas l'existence d'autres zones soumises à l'amplification dans le cancer du sein. Rien ne nous permet d'affirmer que l'on ne découvrira pas d'autres gènes amplifiés, qui viendront s'ajouter à la liste dressée ci-dessus. De plus, on a connaissance d'amplifications sporadiques dans 2 à $5 \%$ des tumeurs analysées. C'est le cas du gène erbB1, qui code pour le récepteur à l'EGF, et des gènes $m y b$ (notre observation), fes [17] et igf1 [18].

\section{Perte ou inactivation de gènes suppresseurs (anti-oncogènes)}

- Les bandes chromosomiques 8 p12 et 10q26 représentent deux

* Positives pour les récepteurs des astrogènes et de la progestérone.
Comme nous l'avons développé dans l'introduction, c'est par l'absence ou l'inactivation de leurs produits que ces gènes concourent à la cancérisation. Cela nécessite logiquement la 


\section{RÉFÉRENCES}

25. Bièche $\mathrm{Y}$, Champème $\mathrm{MH}$, Matifas $\mathrm{F}$, Hacene K, Callahan R, Lidereau R. Loss of heterozygosity on chromosome $7 \mathrm{q}$ and aggressive primary breast cancer. Lance 1992 ; 339 : 139-43.

26. Sato $\mathrm{T}$, Tanigami A, Yamakawa $\mathrm{K}$, et al. Allelotype of breast cancer : cumulative allele losses promote tumor progression in primary breast cancer: Cancer Res 1990 ; 50 : 7184-9.

27. Hollstein M, Sidranski D, Vogelstein B, Harris CC. p53 mutations in human cancers. Science 1991: $253: 49-53$

28. Borrensen AL, Hovig E, SmithSorensen B, et al. Constant denaturant gel electrophoresis as a rapid screening technique for p53 mutations. Proc Natl Acad Sci USA 1991; 88 : 8405-9.

29. Davidoff AM, Humphrey PA, Iglehart DJ, Marks JR. Genetic basis for p53 overexpression in human breast cancer. Proc Nat Acad Sci USA 1991; 88 : 5006-10.

30. Osborne RJ, Merlo GR, Mitsudomi T, et al. Mutations in the p53 gene in primary human breast cancers. Cancer Res $1991 ; 51$ : 6194-8.

31. Runnebaum IG, Mahalakshmi N, Bowman M, Soto D, Sukumar S. Mutations in p53 as potential molecular markers for human breast cancer. Proc Natl Acad Sci USA $1991 ; 88: 10657-61$.

32. Mazars R, Spinardi L, Bencheickh M, Simony-Lafontaine J, Jeanteur P, Theillet C. p53 mutations occur in aggressive breast cancer. Cancer Res 1992; 52 : 3918-29.

33. Lane DP, Benchimol S. p53 : oncogene or anti-oncogene? Genes Dev $1990 ; 4: 1-8$.

34. Horak E, Smith K, Bromley L, et al. Mutant p53, EGF receptor and c-erbB2 expression in human breast cancer. Oncogene $1992 ; 6$ : 2277-84.

35. Thor AD, Moore DH, Edgerton SM et al. Accumulation of $\mathrm{p} 53$ tumor suppressor gene protein : an independent marker of prognosis in breast cancers. J Natl Cancer Inst $1992 ; 84: 845-55$

36. Bourne HR. Suppression with a difference. Science $1991 ; 353$ : 696-8.

37. Chen LC, Kurisu W, Ljung BM, Goldman ES, Moore D, Smith SS. Heterogeneity for allelic loss in human breast can- mise hors service des deux copies du gène dans la cellule tumorale et donc un processus en deux étapes (pour revue, voir [19]) qui a reçu sa consécration expérimentale dans les modèles du rétinoblastome et du néphroblastome.

\section{- Les délétions chromosomiques} Les premières délétions observées dans le cancer du sein l'ont été sur le bras court du chromosome 11, plus particulièrement sur la bande 11 p15 (pour revue, voir [20]). Ces données montraient qu'à l'instar des néphroblastomes et des rhabdomyosarcomes le chromosome $11 \mathrm{p} 15$ pouvait participer au processus tumoral dans le cancer du sein (pour revue, voir [21]). Il est, en fait, rapidement apparu que les délétions et la très probable inactivation d'un gène suppresseur en 11 p15 étaient associées à de nombreux types de cancers [20]. Avec les pertes de matériel observées sur les chromosomes 13q (porteur du gène $R b)[20], 17 \mathrm{p}[20], 1 \mathrm{p}[22], 1 \mathrm{q}$ [20], 3p [20], 17q [23], 18q [23], et $7 q[24]$, ce sont donc à ce jour neuf foyers de délétions qui ont été identifiés dans les cancers du sein (Tableau II). Il faut souligner l'importance toute particulière du chromosome 17 puisqu'il abrite l'antioncogène p53 sur le bras court (17p13.1) et que le gène de prédisposition au cancer héréditaire seinovaire (brcA1) a été localisé sur le bras long (17q21).

Si l'on estime que 60 à $70 \%$ des tumeurs présentent au moins un site chromosomique affecté par un tel événement, il reste à se demander si ces délétions sont bien toutes représentatives de la mise hors service d'un gène suppresseur ou si certaines ne sont pas plutôt le reflet de remaniements aléatoires typiques de l'instabilité génomique qui caractérise les cellules cancéreuses. En fait, la recherche systématique des pertes d'allèles sur deux loci de chaque bras des 22 autosomes [22, 25] a montré que presque toutes les localisations analysées peuvent subir des délétions, mais que certaines peuvent aussi être sujettes à des duplications ou triplications. Comment distinguer alors les événements spécifiques des remaniements aléatoires dans le chaos caryotypique qui accompagne la pro- gression des tumeurs du sein ? Hormis les délétions en $7 q, 11 p$ et $17 p$, qui ont pu être reliées à des paramètres tumoraux de mauvais pronostic, la grande majorité des études ne notent pas d'associations entre délétions et paramètres clinico-pathologiques. Il faut cependant souligner les éléments qui concourent à compliquer l'établissement de corrélations statistiques dans l'étude des délétions. Tout d'abord, la mise en évidence de pertes d'allèles n'est possible que dans les échantillons hétérozygotes (présentant deux allèles) pour les marqueurs étudiés, ce qui, selon le pourcentage d'hétérozygotie au locus considéré, peut réduire sensiblement le nombre de cas informatifs. Cette réduction d'effectif s'aggrave encore lorsque l'on doit cumuler les résultats obtenus avec plusieurs marqueurs. Il en résulte donc que les résultats sont fréquemment obtenus sur des souspopulations tumorales réduites, dont les effectifs permettent difficilement de dégager des statistiques significatives. Il serait donc erroné, ou à tout le moins prématuré, de conclure à l'absence de lien entre délétions chromosomiques et agressivité des tumeurs mammaires. Au contraire, un ensemble d'éléments convergents suggère une telle relation: (1) les tendances (statistiquement non significatives) observées dans les différentes études; (2) les données obtenues sur des tumeurs d'origines anatomiques différentes (le cancer colo-rectal en particulier); (3) les expériences d'hybrides cellulaires (cellules normales et cellules tumorales) où l'introduction de fragments de chromosomes normaux (par fusion de microcellules) dans des cellules tumorales conduit à la régression des caractères tumoraux. Tout cela renforce l'hypothèse de la présence d'anti-oncogènes dans les régions chromosomiques fréquemment délétées dans le cancer du sein.

\section{- Le gène $R b$}

L'archétype des anti-oncogènes, exprimé de façon ubiquitaire et occupant une position centrale dans la régulation du cycle cellulaire, devrait logiquement être fréquemment inactivé dans un large spectre de cancers. Étrangement, cette généralité n'a pas pu se vérifier et les mutations ou les 
réarrangements délétères du gène $R b$ semblent rares dans le cancer du sein. Il en ressort que malgré l'existence de délétions chromosomiques sur le chromosome $13 \mathrm{q}$, où se trouve pourtant $R b$, ce gène est rarement impliqué dans des anomalies structurales détectables par la technique de Southern blot. Toutefois cela n'exclut pas l'inactivation de $R b$ à la suite de mutations plus discrètes (mutations ponctuelles), dont la détection implique l'utilisation de techniques fondées sur la PCR. Si l'on considère la taille et la complexité du gène $R b$, qui s'étend sur $200 \mathrm{~kb}$ et compte 27 exons, on comprend pourquoi ce type d'étude n'a pas fait école. On en reste donc à faire des suppositions sur l'implication de $R b$ dans le cancer du sein, suppositions fondées sur la fréquence de délétions observées en $13 q$ et on peut ainsi estimer que 10 à $15 \%$ des tumeurs du sein sont porteuses d'un gène $R b$ inactivé.

\section{- Le gène $p 53$}

Le gène $p 53$ et son produit accompagnent la recherche en cancérologie moléculaire depuis plus de dix ans. D'abord considéré comme un oncogène, ce n'est que récemment que son potentiel suppresseur de tumeur a été mis en évidence et il apparaît désormais acquis que l'intégrité du gène $p 53$ est atteinte dans tous les types tumoraux étudiés (pour revue, voir [26]) et il semble donc que le développement ou la progression de la majorité des cancers passent par l'inactivation de cet anti-oncogène. La moyenne générale, tous types tumoraux confondus, se place à environ $50 \%$ de tumeurs mutées pour le gène $p 53$ alors que les différentes études en détectent de 13 à $46 \%$ dans les tumeurs du sein [27-32]. Cela placerait donc le cancer du sein dans les types tumoraux à incidence basse ou moyenne, ce qui contraste avec la haute fréquence de délétions $(\geqslant 50 \%)$ enregistrée dans les tumeurs du sein sur le bras court du chromosome 17. Si, de plus, on considère que l'on n'observe pas systématiquement la mutation $\mathrm{du}$ gène $p 53$ dans les tumeurs présentant des pertes alléliques dans la région $17 \mathrm{p} 13.3$, on est tenté de conclure à l'existence d'au moins deux foyers de délétion et peut être de deux anti-

$\mathrm{m} / \mathrm{s} n^{\circ} 9$ vol. 8 , novembre 92

Tableau II

RÉGIONS CHROMOSOMIQUES LES PLUS FRÉQUEMMENT DÉLÉTÉES DANS LE CANCER DU SEIN

\begin{tabular}{|c|c|c|c|c|}
\hline \multirow{2}{*}{$\begin{array}{l}\text { Localisation } \\
\text { chromosomique }\end{array}$} & \multirow[t]{2}{*}{ Fréquence } & \multirow{2}{*}{$\begin{array}{c}\text { Gène suppresseur } \\
\text { connu } \\
\text { dans la région }\end{array}$} & \multicolumn{2}{|c|}{ Association avec } \\
\hline & & & délétions & amplifications \\
\hline $1 p$ & 9 à $41 \%$ & $?$ & & \\
\hline $1 q$ & 9 à $30 \%$ & $?$ & & \\
\hline $3 p$ & 19 à $35 \%$ & $r c c$ & $11 p, 17 p$ & \\
\hline $6 q$ & 9 à $48 \%$ & $?$ & & \\
\hline $7 q$ & $40,5 \%$ & ? & & \\
\hline $11 p$ & 10 à $27 \%$ & $w t 2$ & $\begin{array}{l}3 p, 13 q, 17 p \\
17 q, 18 q\end{array}$ & erbB2, myc, int 2 \\
\hline $\begin{array}{l}13 q \\
16 q\end{array}$ & $\begin{array}{c}21 \text { à } 28 \% \\
45 \%\end{array}$ & $\begin{array}{c}R b \\
?\end{array}$ & $11 \mathrm{p}$ & \\
\hline $17 p$ & 20 à $61 \%$ & p53 & $3 p, 11 p, 18 q$ & erbB2 \\
\hline $17 q$ & 10 à $39 \%$ & $n f 1$, brcAl & $11 p$ & \\
\hline $18 q$ & 8 à $69 \%$ & $d c c$ & $11 p, 17 p$ & \\
\hline
\end{tabular}

oncogènes sur le bras court du 17 $[29,31]$.

Une autre voie d'approche (très complémentaire de l'approche moléculaire) des anomalies p53 dans les tumeurs humaines est fournie par l'analyse de l'accumulation de la protéine p53 à l'aide de méthodes immunologiques (immunoprécipitation, Western blot ou immunohistochimie). Cette approche repose sur la plus grande stabilité de la protéine p53 mutée, qui la rend détectable par immunohistochimie alors que la forme normale ne l'est pas. Cela a engendré l'hypothèse d'un signal immunologique positif avec un anticorps anti-p53 signalant un allèle mutant [33]. On voit donc tout l'avantage de pouvoir détecter in situ une anomalie p53 par immunohistochimie. La méthode est plus simple, peut être pratiquée sur des biopsies de petite taille et peut éventuellement détecter des micro-foyers positifs dans des structures largement négatives. Toutefois, on note une certaine hétérogénéité dans les résultats, autant par les pourcentages de tumeurs positives que par les types de marquage. A cela s'ajoute une certaine variabilité en fonction de l'anticorps utilisé et l'absence de marquage en cas de mutation non-sens. On voit donc que l'immunocytomarquage présente quelques limites, mais, à l'inverse, la détection directe des mutations après PCR n'est pas non plus sans défaut. En effet, dans les tumeurs présentant une forte hétérogénéité cellulaire, le signal mutant pourra être obscurci par le signal normal et ainsi aboutir à manquer certaines mutations. Il sera donc certainement très intéressant de procéder à des comparaisons systématiques des deux méthodes pour répondre à cette question.

La faible taille des échantillons analysés jusqu'ici n'autorise pas de conclusions définitives sur les corrélations entre les anomalies de la p53 et les paramètres cliniques et pathologiques. On notera toutefois trois études qui tendent à relier ces anomalies à des caractères généralement associés aux tumeurs évoluées. La première observe une corrélation positive entre la détection immunohistochimique de la p53 et la surexpression d'erbB1 et d'erbB2 [34]. La deuxième relie la présence de mutations au niveau du gène à l'absence de récepteurs hormonaux [31] et suggère que ces mutations seraient des événements tardifs, plus en relation avec la progression tumorale qu'avec son initiation. La troisième, outre une association avec l'absence de récepteurs hormonaux et un grade nucléaire élevé, montre une corrélation entre la détection immunohistochimique de la p53 et un risque accru de rechute et/ou de décès avant cinq ans [35].

En conclusion de cette section sur les gènes suppresseurs de tumeur, on notera le grand nombre de gènes potentiellement en cause et donc la diversité des combinaisons possibles. 
En effet on dispose, à l'heure actuelle, d'éléments qui suggèrent que certaines mutations pourraient aussi s'exprimer selon un mode dominant négatif. Supposons que la mutation ait pour effet la perte de l'activité normale de la protéine, mais que la forme mutante (inactive) entre en compétition avec la forme normale pour son substrat ou encore se multimérise avec sa contrepartie normale, on assistera alors à la formation de complexes inactifs dans les cellules porteuses d'une mutation. La présence de la forme mutante empêcherait le fonctionnement du produit de l'allèle sauvage et a été définie comme un modèle d'expression dominant négatif. Ce type de mécanisme a été effectivement proposé pour certaines mutations p53 mais aussi pour le gène fap/apc [36]. Ainsi, d'après ce schéma, un allèle muté peut s'exprimer même en présence de l'allèle sauvage ; toutefois, on conçoit l'avantage que procure la perte de la version normale, même dans un système comme celui-ci. En effet, on ne peut exclure un effet de dose, où quelques molécules normales échappent aux complexes inactifs et réussissent, malgré tout, à exercer leur effet négatif sur la prolifération cellulaire.

\section{Vers une carte de la progression tumorale dans le cancer du sein ?}

Une fois recensées, encore la liste n'en est-elle certainement pas exhaustive, toutes les altérations génomiques observées dans le cancer du sein, il importerait de savoir les ordonner de façon chronologique selon un schéma de progression tumorale. Malheureusement, le cancer du sein est bien différent cliniquement de celui du côlon en ce qu'il n'est pas possible de décrire un tel schéma clinique ou anatomo-pathologique sur lequel poser des jalons moléculaires, en raison notamment de notre incapacité à identifier des lésions précancéreuses. C'est dans ce but que plusieurs équipes travaillent à la mise en évidence de caractères permettant d'identifier les mastoses à risque. On se borne dès lors à tenter de corréler entre eux les différents événements génomiques (amplifications/délétions/mutations) répertoire particulièrement varié. On retiendra des associations entre les amplifications de myc et d'erbB2 [2], les amplifications en $11 \mathrm{q} 13$ et en 8p12 (figure 2) (Tableau I) [16] et de possibles réseaux associatifs impliquant des pertes de matériel génétique centrées autour des délétions en $11 p$ (associations $11 p-3 p, 11 p-13 q$, $11 \mathrm{p}-17 \mathrm{p}, 11 \mathrm{p}-17 \mathrm{q}, 11 \mathrm{p}-18 \mathrm{q})$ et en $17 \mathrm{p}$ (associations $17 \mathrm{p}-3 \mathrm{p}, 17 \mathrm{p}-11 \mathrm{p}$, 17p-18q) (Tableau II) [22, 23, 25]. Il faut reconnaître que l'image reste encore assez floue et que seule l'identification des anomalies survenant précocement dans le cancer du sein nous permettra de la clarifier. On remarquera toutefois l'intéressante observation de Chen et al. [37] qui, analysant une même série de tumeurs du sein avec des sondes spécifiques de 12 bras chromosomiques, observèrent dans les tumeurs présentant des pertes d'allèles concomitantes en plusieurs sites, que les niveaux de réduction alléliques n'étaient pas identiques pour toutes les sondes. Ainsi, une tumeur pouvait présenter une perte totale sur le $17 \mathrm{p}$, alors que la délétion sur le chromosome 22 n'était que partielle. D'autres combinaisons n'étaient pas exclues, telles que délétion totale en $3 p$ et partielle en 18q. Ces données donnent un aperçu de la chronologie d'apparition de ces anomalies, mais le nombre important de combinaisons possibles imposerait une analyse sur un très grand échantillonnage pour en tirer une image claire. D'autres perspectives nous seront offertes par l'utilisation, en conjonction avec les techniques désormais classiques telles que le Southern blot et la PCR, de l'hybridation in situ sur chromosome en interphase à l'aide de sondes fluorescentes. Grâce à cette technique, il sera possible d'accéder à la structure du génome au niveau de cellules individuelles (ou de groupes de cellules) au sein d'une tumeur, d'y détecter les niveaux d'amplification d'un ou de plusieurs gènes (en utilisant des fluorochromes différents), de distinguer les amplifications vraies des déséquilibres de ploïdie et de vérifier l'intégrité de chromosomes d'intérêt. La généralisation de cette méthode se conjugue au présent et on peut parier qu'elle sera transférée à la pratique clinique dans un avenir peu éloigné

\section{Summary}

Acquired genetic alterations in human breast cancer

Recent evidence led to consider the evolution of a tumor from a premalignant to a fully transformed phenotype as a linear progression where each step correspond to the onset of a genetic anomaly. Mutations affecting oncogenes lead to their activation, whereas those hitting anti-oncogenes cause the loss of their anti-proliferative potential. Therefore, each mutation drives the cell further into anaplasia and in the end the most evolved the cancer cells are, the greater the number of alterations they bear. Within the last few years there has been a systematic effort to establish the inventory of genetic alterations in human tumors, the aim being to sketch out a genetic map of cancer. Human breast cancer, being a leading cause of cancer mortality for women in Western Europe and North America, has focused the attention very early on. It bears a whole range of anomalies including amplification at 5 different loci and loss of genetic material involving 10 chromosomal sites, to which mutations in the p53 gene must be added. Thus, potential combinations of oncogene activation and anti-oncogene loss are multiple and it will be important to have a better knowledge of their sequential order of occurrence. This, indeed, should give us more insight on each step of breast cancer evolution. It could then be possible to correlate specific sets of alterations with a particular clinical stage or disease pattern. Thus, genotyping of breast tumors should prove usefull in the clinical management by helping establish diagnosis and evaluate the prognosis of a tumor. Furthermore the field is evolving from making the inventory to mapping the altered chromosomal regions with the ultimate aim to identify and clone the genes involved. This should teach us as much on the mechanism of tumorigenesis as on genome structure and organisation. 